\title{
ESSENTIAL SHRINKAGE OF THE CONJUNCTIVA IN EPIDERMOLYSIS BULLOSA HEREDITARIA*
}

\author{
BY
}

\author{
MAHMUD ALI SHAH AND MUBARIKA SHAH \\ From the Departments of Ophthalmology and Paediatrics, Dow Medical College, and \\ Civil Hospital, Karachi, Pakistan
}

EPIDERMOLYSIS bullosa is a rare hereditary disease, appearing at variable times after birth, in which, for reasons not yet clearly understood, bullae appear on the skin with, and sometimes without, the slightest trauma. Ocular involvement is extremely rare. Parts of the eye reported to be affected are the eyelids (Tobias, 1928), the cornea (Pernet, 1904; de Schweinitz, 1913; Ziegler, 1913; Montpellier and Lacroix, 1920), and the conjunctiva. Involvement of the conjunctiva ultimately leads to its essential shrinkage, but of this very few cases are on record. Cohen and Sulzberger (1935) could collect only four cases in the ophthalmological and dermatological literature, and Sorsby and others (1951), reviewing the relevant literature, brought the number of reported cases to six, and presented two cases of their own as vesicular affections allied to epidermolysis bullosa.

The findings in the case reported below are compatible with epidermolysis bullosa hereditaria, involving the cornea and causing essential shrinkage of the conjunctiva. The patient's family tree is shown in Fig. 1.



Fig. 1.-Family tree.
A. Died of fever.
B. Died of diarrhoea and vomiting.
C. Case reported in full. D. Died of kidney trouble; blind (? due to
measles).
E. Died of small pox.
G. Blind (cause not known).
H. Moderately myopic.
I. Freckles on nose and cheeks.
J. Died of diarrhoea
K. Died of vomiting
L. Puberty goitre.
F. Died of small pox.

*Received for publication March 21, 1955. 


\section{Case Report}

A married woman aged 35 was admitted to the Civil Hospital, Karachi, on December 1, 1950, with symblepharon, for reconstruction of the inferior conjunctival fornices of both eyes. There was a peculiar dark- to light-brown mottling of the skin of the lids, face, and body. The history of the skin lesion and eye condition dated from infancy.

Family History.-The patient's mother, aged 65, was stated to be alive and healthy, and the father was reported to have been free from any significant disease. There was no consanguinity. The ten children born of this union could be divided into two groups; those who were apparently healthy, and those who exhibited skin lesions (Fig. 1):

(1) The first group (II, 1-5) comprised five children which were all healthy. Two males died in infancy and the offspring of the rest were stated to be healthy except those of one female aged 38 (II, 5), who was married to a healthy male with no consanguinity. Of their seven children (III, 7-13), two females showed marked pigmentation of the skin of the hands and feet, which was thickened and ridged, loose irregular teeth, and bieeding gums. One of these two, in addition, had chronic suppurative otitis media, chronic trachoma, and history of discrete bullae on the legs and feet. Some of these bullae were septic and appeared as granulomatous ulcers. Investigations were not permitted of any member of this group.

(2) The second group (II, 6-10) also comprised five members, all of whom were reported to have been suffering from a vesicular affection of the skin. One female aged 35 (II, 6), who forms the subject of this report, is married (nonconsanguineously) and has two daughters (III, 14 and 15) who are both healthy.

Clinical History.-The patient started to have " blisters" on her skin from the third day after birth. The blisters, which were filled with clear fluid, appeared first on the ankles, later spreading to leg, forearm, and then to the rest of the body. They came in crops, each crop lasting 8 to 15 days. On healing they left pigmented scars. As far as could be ascertained there was no association of injury with the production of blisters. The interval between the recurrence increased progressively from 2 or 3 months to 2 years, the patient remaining comparatively free from blisters in the intervening period. No blisters have appeared since the age of 15 , but the patient thinks she is more prone than others to minor injuries becoming septic. There is itching all over her body in winter months, but chiefly on the arms and legs. There is vague history of occasional difficulty in swallowing for "a number of" years. Other points of interest are:

(i) Delayed dentition, first tooth appearing at the age of 15 months.

(ii) "Sore eyes" from " childhood" at irregular intervals.

(iii) Operation on both eyes at the age of 14 for "a membrane growing on the eyes" (? false pterygia), but the lower lids have been stuck to the eyeballs since childhood.

(iv) Gums spongy and teeth loose "very early"; by the age of 15 she was edentulous, some teeth having fallen out, and others having been extracted.

(v) Measles at the age of 4 years.

Physical Examination.-Average build, fairly good general health, edentulous.

(i) Skin.-Generally thin and atrophic, stretched and glossy over the palms. The lip margins are ill defined. The whole body shows light- to dark-brown pigmentation (hair partly grey, nails within average limits, no milia).

(ii) Eyes.-Palpebral fissure narrower than normal. Upper lid margin crosses the cornea about the middle in both eyes. The skin of the lids shows light-brown mottling. Palpebral conjunctiva of upper lids shows superficial scarring over papillary hypertrophy. Superior fornix shows a few trachoma follicles (upper half of bulbar conjunctiva appears normal). Inferior fornix in both eyes obliterated by dense scar tissue (Fig. 2). Corneae show regressive trachomatous pannus in the upper third, sparsely vascularized superficial opacities in the lower quarter, extending from about 3 to 9 o'clock, and multiple nebulae elsewhere. Vision reduced to $6 / 36$ in both eyes. Interior as far as $c$ an be examined appears within average limits. 
FIG. 2.-Scar tissue in inferior fornices.

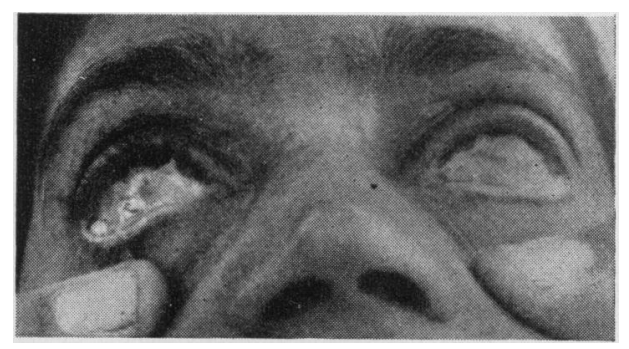

\section{Investigations}

Laboratory Findings.-Essentially negative. Results of uroporphyrins and coproporphyrins inconclusive. Radiological studies of skull showed the sella turcica within normal limits. Skiagrams after barium meal revealed no organic cause of dysphagia. Iodides internally (as suggested by Cohen and Sulzberger, 1935) had no effect. Patch test with potassium iodide and potassium bromide negative.

Skin.-Sections from outer part of right arm and right lower lid was reported on by Dr. H. Haber with the corroboration of Dr. John O. Oliver (Department of Pathology, St. John's Hospital for the Diseases of the Skin, London):

Eyelid (Fig. $3 a$ and $b$, overleaf).-The epidermis is thin and the epidermo-dermal junction is flattened, in keeping with the normal histology of the eyelid. The striking feature is a subepidermal oedema, patchy slit formation and total detachment of the epidermis from the underlying corium in several places. Staining for elastica reveals an absence of the subepidermal garland and of elastica fibres at the stratum papillare and subpapillare. Solar elastosis arranged round follicles and extra follicular patches can also be demonstrated. There is no inflammatory response noticeable within the corium. The histology is in keeping with epidermolysis bullosa hereditaria.

Arm (Fig. $4 a$ and $b$, overleaf).-The epidermis shows hyperkeratosis, atrophy, and acanthosis. The epidermo-dermal junction is flattened with oedema and slit formation in several places. There is considerable pigmentary incontinence demonstrable which might be due to the disturbance at the epidermo-dermal junction. The subepidermal garland is absent and the stratum subpapillare reveals the elastica to consist of the thick dark blue blocks. The midcutis shows normal elastica fibres. There is no appreciable inflammatory response demonstrable. The histology is compatible with epidermolysis bullosa hereditaria.

Treatment.-Symblepharon was corrected by conjunctivoplasty on January 9, 1951, with fairly satisfactory results (Fig. 5, overleaf).

\section{Discussion}

The hereditary nature of the affection, the skin, and conjunctival lesions, and the histopathological findings of the skin suggest the diagnosis. It will be noted that there is subepidermal slit formation in contradistinction to intra-epidermal slits as in pemphigus.

Ocular findings, except for the symblepharon and the opacities in the lower part of the corneae, suggest chronic trachoma. Symblepharon would be the logical result of the bullae on the conjunctiva but why this should have been limited only to the inferior fornices is not clear. In view of the history of operation, on both eyes, 20 years ago, the corneal opacities could be attributed to false pterygia, as a direct consequence of bullae on the conjunctiva and the cornea.

Clinically, epidermolysis bullosa has been divided into the simple and 


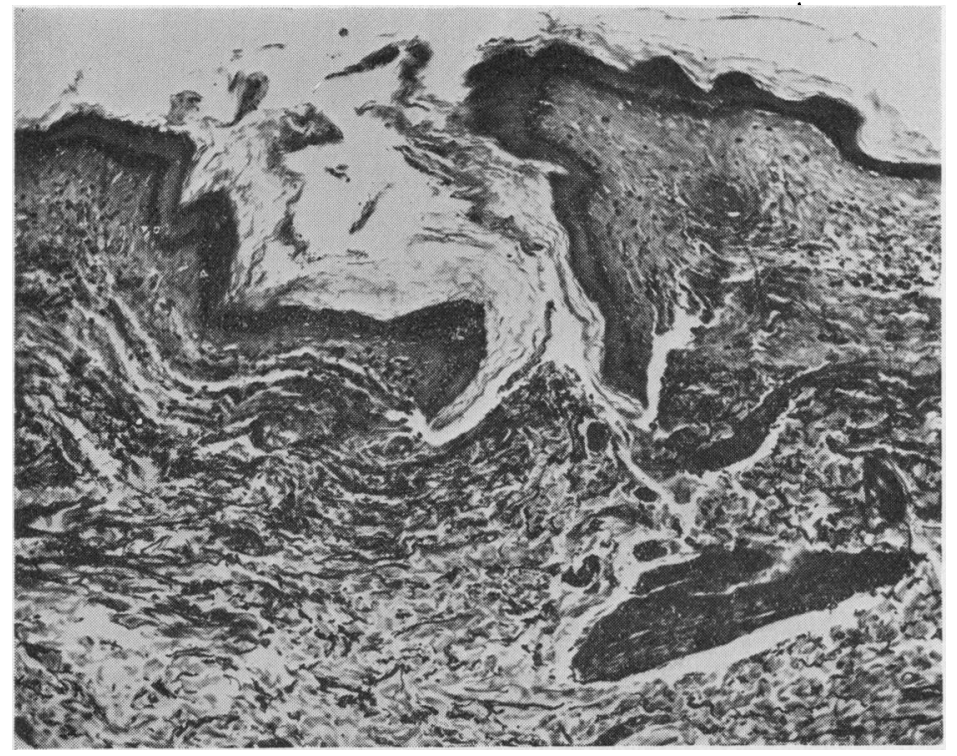

FIG. 3(a).-

Biopsy of eyelid.

Fig. 3(b).-Biopsy of eyelid.

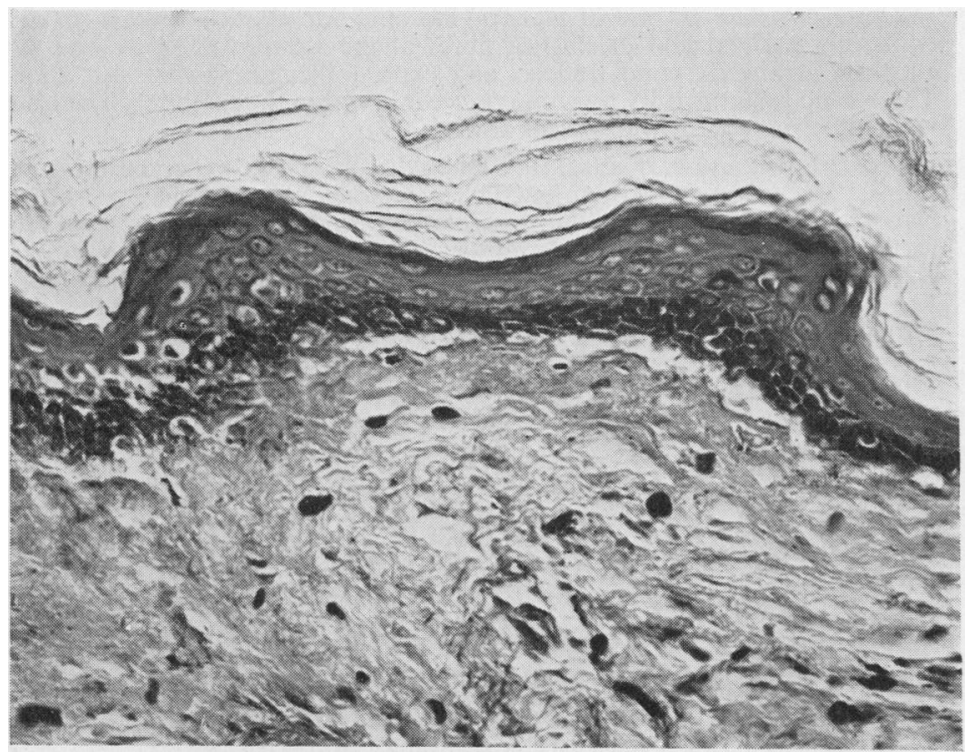

dystrophic varieties. It is difficult to fit the case reported into either of these forms as it shows some features of each. The good health of the patient, the normal hair and nails, the absence of epidermal cysts, and the fact that the disease has in time burnt out, point to its being of the simple variety. On the other hand, the reported appearance of bullae soon after birth, the generalized distribution, the abnormality and early decay of teeth, and the 


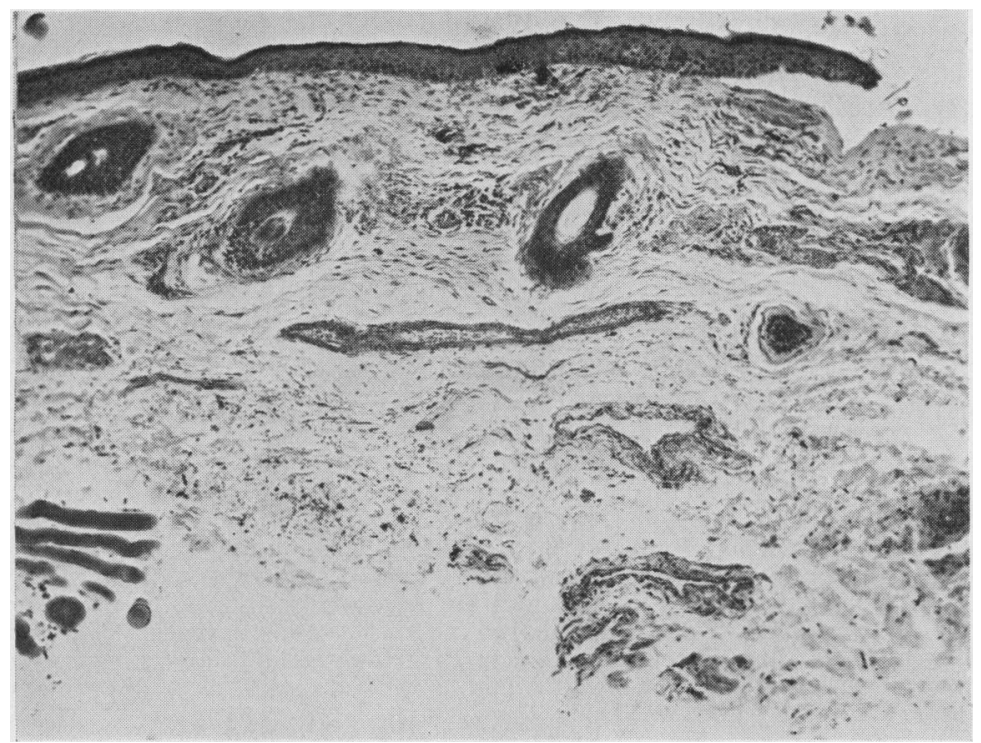

FIG. 4(a).-

Biopsy of

skin of arm.



Fig. 5.-Palpebral tissue after correction of symblepharon.

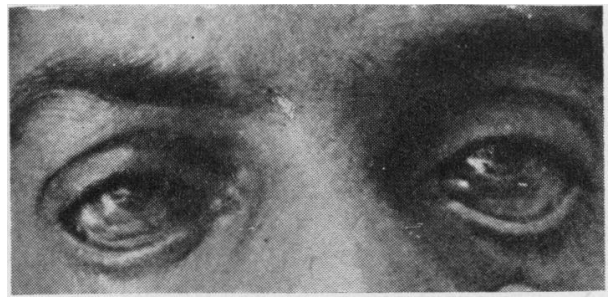


involvement of the conjunctiva and possibly of the cornea with scarring, are features usually attributed to the dystrophic variety.

It is not possible to make a final assessment of the mode of inheritance of the disease. Some members of the family could not be examined and the pedigree is limited to two generations. From the evidence available irregular dominance would appear to be suggested.

\section{Summary}

A study is presented of a case of essential shrinkage of conjunctiva in which the findings were compatible with epidermolysis bullosa hereditaria.

We wish to thank Prof. Arnold Sorsby for help in having the biopsy slides examined and for elucidating some points in genetics, Doctors $\mathbf{H}$. Haber and John O. Oliver for their expert histopathological report on skin biopsies, Prof. Parker Heath for much valuable instruction and discussion during the period that one of us (M.A.S.) worked in his laboratory at Boston, Mass.; Dr. Violet E. Hawkinson (Minneapolis) for suggestions and references, and for making available standards for estimating porphyrins in the urine, and Dr. Abrar Hussain (Edinburgh) and the photographic department of the University of Edinburgh for the photomicrographs. The willing cooperation received from the Departments of Clinical Pathology and Radiology of the Civil Hospital, Karachi, and from the technical staff of the Pathology Department of the Dow Medical College, Karachi, is also gratefully acknowledged.

\section{REFERENCES}

Cohen, M., and Sulzberger, M. B. (1935). Arch. Ophthal. (Chicago), 13, 374.

MontPellier, J., and LaCrolX, A. (1920). Ann. Derm. Syph. (Paris), be ser, 1, 575.

PfRNeT, G. (1904). Ophthalmoscope, 2, 308.

SCHWEINITZ, G. E. DE (1913). Trans. Coll. Phys. Philad., 35, 417. Quoted by Sorsby and others (1951).

Sorsby, A., RoBerts, J. A. F., and Brain, R. T. (1951). Docum. ophthal., 5, 118.

TOBIAS, N. (1928). Arch. Derm. Syph. (Chicago), 18, 224. Quoted by Sorsby and others (1951).

Ziegler (1913). Trans. coll. Phys. Philad., 35, 418. Quoted by Sorsby and others (1951). 